\title{
Generalized Dowling Degos disease: A rare genetic disorder
}

\author{
Saurabh Sharma ${ }^{1, *}$, Simplepreet Kaur ${ }^{2}$, Roopam Bassi ${ }^{3}$ \\ ${ }^{1,3}$ Professor, ${ }^{2}$ Junior Resident, ${ }^{1,2}$ Dept. of Dermatology, ${ }^{3}$ Dept. of Physiology, Sri Guru Ram Das Institute Of Medical Sciences \\ and Research, Amritsar, Punjab, India \\ *Corresponding Author: \\ Email: drsaurabhsharma@yahoo.co.in
}

\begin{abstract}
Dowling Degos disease (DDD) is an uncommon, autosomal dominant genodermatosis characterized by acquired, flexural hyperpigmentation. Clinico-histopathological correlation is diagnostic showing multiple hyperpigmented macules clinically and marked, heavily pigmented, slender and elongated rete ridges on histology. This entity needs to be differentiated from other reticulate hyperpigmentary disorders. Due to rarity of this condition, we report a case of generalized Dowling Degos disease in an adolescent female.
\end{abstract}

Keywords: Dowling Degos disease, Genodermatosis, Hyperpigmentation.

\section{Introduction}

Dowling Degos Disease (DDD) is a rare genetic disorder which is inherited as an autosomal dominant trait. It is considered to be a part of reticulate hyperpigmentary genodermatosis and is characterized by presence of numerous, symmetrical and progressive hyperpigmented macules located over face, neck, axillae, trunk, groins and flexural aspect of extremities. Some of the macules may coalesce together to form a reticulate pattern. Other noteworthy features include presence of comedo like lesions and pitted acneiform scars. This benign condition has tendency to appear and/or worsen during puberty. ${ }^{1}$ The genetic abnormalities have been attributed to mutations and deletions in keratin 5 gene and a gene locus mapping to chromosome $17 \mathrm{p} 13.3 .^{2,3}$ Due to rarity of this disorder, we report a case of generalized DDD in an adolescent female.

\section{Case Report}

A 19 year old female patient presented to the dermatology outpatient department with complaint of presence of multiple, brown colored, small lesions over face, neck, axillae, trunk and extremities. Lesions started at the age of 4 years over neck and gradually progressed to involve the other areas. Patient revealed the exacerbation of lesions after puberty. Lesions were asymptomatic and not associated with any inflammatory condition. On clinical examination, there were multiple, bilaterally symmetrical, hyperpigmented macules ranging in color from light to dark brown, present over face, neck, chest, axillae, abdomen, back, forearms, groins and thighs with sparing of distal part of upper and lower extremities, palms and soles (Fig. 1). Over face, macules were more concentrated around periorbital and perioral area (Fig. 2). Periorbital area also showed presence of multiple atrophic pitted scars along with few comedone like follicular papules (Fig. 3). On systemic examination, no abnormality was detected. There was no history of similar lesions in any other family member. The routine investigations including complete blood count, urine complete examination, liver function test and renal function tests, revealed no abnormality. For histopathological examination, a punch biopsy of size $3 \mathrm{~mm}$, was performed from hyperpigmented macule over thigh. Biopsy revealed hyperkeratosis with presence of epidermal acanthosis, elongation of rete ridges and increased melanin in basal layer cells especially at the tips of rete ridges. At few places, pigmentary incontinence was also observed (Fig. 4). On the basis of clinical and histopathological findings, diagnosis of DDD was confirmed.

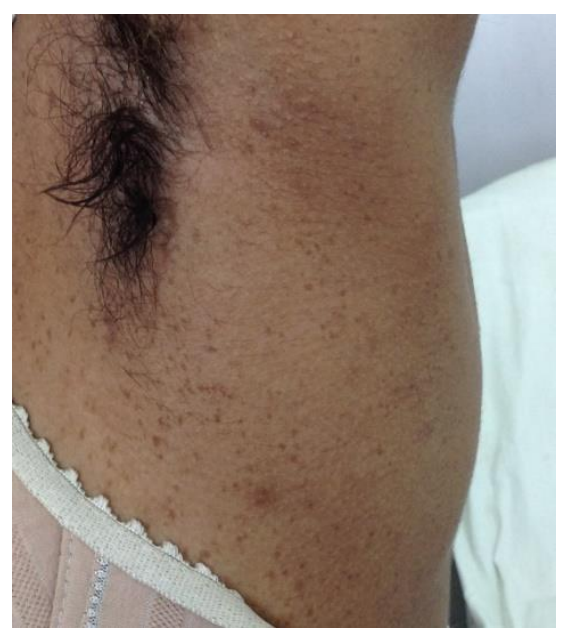

Fig. 1: Hyperpigmented macules present in left axilla 

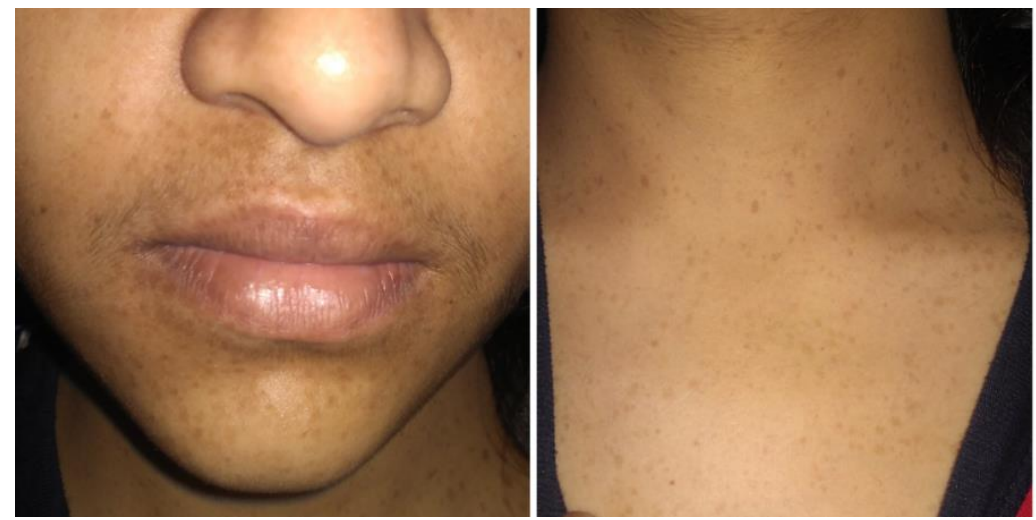

Fig. 2: Hyperpigmented macules present over perioral area and neck

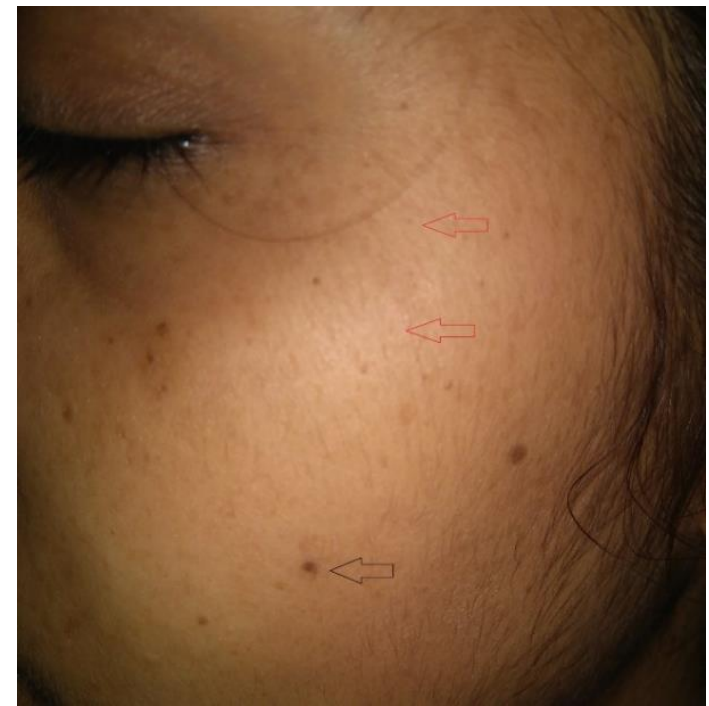

Fig. 3: Multiple pits in the periorbital area (red arrows) with comedo like follicular papule (black arrow)

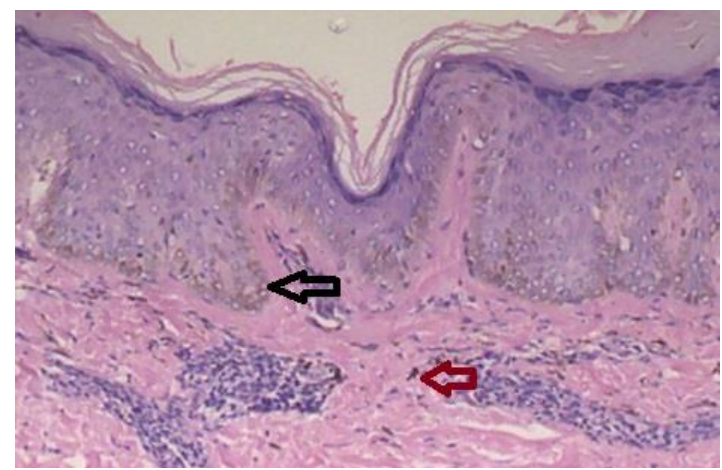

Fig. 4: Histopathology showing hyperkeratosis with epidermal acanthosis, elongation of rete ridges with increased melanin in basal layer (black arrow) and pigmentary incontinence (red arrow). (H \& E stain, 400X)

\section{Discussion}

In present case, other differentials considered were reticulate acropigmentation of Kitamura (RAPK), dermatopathia pigmentosa reticularis (DPR), dyschromatosis hereditaria universalis (DHU). RAPK is characterized by presence of atrophic pigmented spots on the back of the hands and feet and palmar pits. DHU is characterized by diffuse symmetrically distributed hypopigmented macules mixed with hyperpigmentation. DPR can be distinguished by the presence of palmoplantar keratoderma with punctiform accentuation, nail and eye changes. ${ }^{4}$ None of the findings consistent with these three differentials were observed in our patient. Clinico-histopathological correlation helped in confirming the diagnosis of DDD.

DDD was first described in 1938 by Dowling and Freudenthal and by Degos in $1954 .{ }^{5}$ More commonly, it is inherited as an autosomal dominant mutation, but it can also occur sporadically. ${ }^{6}$ There is no racial predisposition, however, there may be a predilection for females. ${ }^{2}$ The disease results from a loss of function mutation in the keratin 5 gene (KRT5) and defect in the gene locus mapping to chromosome $17 \mathrm{p} 13.3$ and chromosome $1 \mathrm{q} 21$. $^{2,3}$

In generalized DDD, sites involved are predominantly flexures; axillae, groins, intergluteal and inframammary folds, anticubital fossa, neck. Scalp, face and arms involvement is also seen. ${ }^{1}$ Presence of multiple pitted facial scars particularly over perioral areas has been documented in literature. ${ }^{7}$ In the present case, multiple pitted facial scars were present but not typically around perioral area rather scars were more concentrated over periorbital area. There is a previous report stating involvement of vulva in a female with DDD. ${ }^{8}$ Few reports have been published revealing coexistence of DDD and RAPK.

Due to overlap of clinical features, differentiation between reticulate hyperpigmentary conditions can be difficult sometimes. Histopathological features act as diagnostic tool for differentiating DDD from other conditions. Presence of acanthosis, downward elongation and thinning of rete ridges with concentration of melanin at rete ridge tips are typical of DDD. ${ }^{9}$ In our case, we observed hyperkeratosis, acanthosis, elongation of rete ridges but without thinning and increased melanin pigment at basal layer. Galli-Galli disease is a variant of DDD where 
acantholysis is the characteristic feature on histopathology. ${ }^{10}$ In present case, no acantholysis was observed, hence Galli-Galli disease was ruled out.

Counselling regarding the benign nature of disease forms an integral part of management of this condition. Other treatment modalities that can be used are topical and systemic retinoids, lasers like Q-switched NdYAG, alexandrite. None of the available treatment options have been proved satisfactory till date. ${ }^{11}$ In present case, patient was counselled regarding the disease and available treatment modalities.

\section{Conflict of Interest: Nil.}

\section{References}

1. Wititsuwannakul J and Noppakun N. Generalized Dowling- Degos Disease: Case Reports. Ann Dermatol. 2013;25(3):360-364.

2. Liao H, Zhao Y, Baty DU, et al. A heterozygous frameshift mutation in the V1 domain of keratin 5 in a family with Dowling-Degos disease. J Invest Dermatol. 2007;127(2):298-300.

3. Li CR, Xing QH, Li M, Qin W, Yue XZ, Zhang XJ, et al. A gene locus responsible for reticulate pigmented anomaly of the flexures maps to chromosome 17p13.3. J Invest Dermatol. 2006;126:1297-301.

4. R.E. Schnur, W.R. Heymann. Reticulate hyperpigmentation. Semin Cutan Med Surg. 1997;16:7280.
5. Kumar AS, Pandhi RK, Jacob M. Dowling-Degos disease. Indian J Dermatol Venereol Leprol. 1986;52:4851.

6. Crovato F, Nazzari G, Rebora A. Dowling-Degos disease (reticulate pigmented anomaly of the flexures) is an autosomal dominant condition. Br J Derm. 1983;108:473476.

7. Zimmermann CC, Sforza D, Macedo PM, AzulayAbulafia L, Alves Mde F, Carneiro SC. Dowling-Degos disease: classic clinical and histopathological presentation. An Bras Dermatol. 2011;86(5):979-82.

8. Horner M E, Parkinson K E, Kaye V, Lynch P J. Dowling-Degos Disease involving the vulva and back: Case report and review of the literature. Dermatol Online J. 2011;17(7):1.

9. Rathoriya SG, Soni SS, A sati D. Dowling-Degos disease with reticulate acropigmentation of Kitamura: Extended spectrum of a single entity. Indian Dermatol Online J. 2016;7:32-5.

10. Gomes J, Labareda J, Viana I. Galli-galli disease: a rare acantholytic variant of dowling-degos disease. Case reports in medicine. 2011;2011.

11. Vasudevan B, Verma R, B adwal S, Pragasam V, Moorchung N, Badad A. A case of reticulate acropigmentation of Kitamura: Dowling Degos disease overlap with unusual clinical manifestations. Indian $J$ Dermatol. 2014;59:290-2.

How to cite this article: Sharma S, Kaur S, Bassi R. Generalized Dowling Degos disease: A rare genetic disorder. Ind $\mathrm{J}$ Clin Exp Dermatol. 2018;4(3):260-262. 